\title{
Implementing Query Completeness Reasoning
}

\author{
Werner Nutt \\ Free Univ. of Bozen-Bolzano \\ Bozen, Italy \\ werner.nutt@unibz.it
}

\author{
Sergey Paramonov
KU Leuven \\ Leuven, Belgium \\ sergey.paramonov@kuleuven.be
}

\author{
Ognjen Savković \\ Free Univ. of Bozen-Bolzano \\ Bozen, Italy \\ ognjen.savkovic@unibz.it
}

\begin{abstract}
Data completeness is commonly regarded as one of the key aspects of data quality. With this paper we make two main contributions: (i) we develop techniques to reason about the completeness of a query answer over a partially complete database, taking into account constraints that hold over the database, and (ii) we implement them by an encoding into logic programming paradigms. As constraints we consider primary and foreign keys as well as finite domain constraints. In this way we can identify more situations in which a query is complete than was possible with previous work. For each combination of constraints, we establish characterizations of the completeness reasoning and we show how to translate them into logic programs. As a proof of concept we ran our encodings against test cases that capture characteristics of a real-world scenario.
\end{abstract}

\section{Categories and Subject Descriptors}

H.2.7 [Database Management]: Database Administration-Repository, Data warehouse, Security, integrity, and protection

\section{Keywords}

Data Quality; Data Completeness; Answer Set Programming

\section{INTRODUCTION}

In database applications such as information integration and decision support, one is interested in data sets that are complete, in the sense that the data represent all relevant facts that hold in the real world. In many situations, though, it is only possible to guarantee partial completeness of the data, which means that for certain aspects of the application domain the data are complete, but not for others. In such a situation, one would like to know at least whether the available data are sufficient to answer a given query completely, that is, whether the answers to the query over the available data are the same as if the data set were complete $[5,6,8,9]$.

School Information System. A typical scenario is the management of administrative data that are contributed by people. An example is the management of school data in the province of Bolzano,

Permission to make digital or hard copies of all or part of this work for personal or classroom use is granted without fee provided that copies are not made or distributed for profit or commercial advantage and that copies bear this notice and the full citation on the first page. Copyrights for components of this work owned by others than ACM must be honored. Abstracting with credit is permitted. To copy otherwise, or republish, to post on servers or to redistribute to lists, requires prior specific permission and/or a fee. Request permissions from Permissions@ acm.org.

CIKM'15, October 19-23, 2015, Melbourne, Australia.

(C) 2015 ACM. ISBN 978-1-4503-3794-6/15/10 ...\$15.00.

DOI: http://dx.doi.org/10.1145/2806416.2806439. which motivated the work reported here. Data in the school information system of the province are often incomplete because each school is individually responsible for inserting its data into the system and because for certain kinds of data the contribution is optional. Decision makers, however, need to know whether or not the statistics on which they base the allocation of resources to schools are derived from complete data. As often some parts of the data are complete, one would like to automatically provide guarantees that some query answers are guarantees and hint at those that need further checks.

Example. Consider the following example of a partially complete school database. Among other data, the database contains facts about pupils and classes, e.g., "John belongs to class $a 1$ at Newton school", pupil(john,al,newton), or "Class al at Newton school is at level 1 and it belongs to the science branch", $\operatorname{class}(a 1$, newton, 1, sci).

Assume we know that we have all pupils from class $a l$ and all pupils from class $b 2$ at Newton school. Thus, the query that asks for "all pupils in class $a 1$ at Newton school", $Q_{\mathrm{a} 1 \mathrm{n}}(N) \leftarrow \operatorname{pupil}(N, a 1$, newton), has a complete answer. However, if we consider the query that asks for "all science pupils at Newton school", $Q_{\mathrm{sn}}(N) \leftarrow$ $\operatorname{pupil}(N, C$, newton $), \operatorname{class}(C$, newton, $L$, sci $)$, then under the completeness assumptions above we cannot say whether the query answer of $Q_{\mathrm{sn}}$ is complete. Note that, in principle, there can be other classes different from $a 1$ and $b 2$.

Databases typically come with integrity constraints such as primary and foreign keys. But there can be also other kinds of constraints that restrict the domains of attributes.

Example (cont'd). Continuing the example, assume that there is a constraint on the class relation, saying that "if a class at Newton school belongs to the science branch then that class has a class code among al and b2", expressed with class $\{$ school = newton, branch $=s c i\}[$ ccode $]=\{a 1, b 2\}$. We call this a conditional finite domain constraint.

Now we can reason in the following way. The query $Q_{\text {sn }}$ selects Newton pupils from the science branch. Since the above constraint holds for the class relation, and pupil is joined with class, pupils in $Q_{\mathrm{sn}}$ must be either from class $a 1$ or $b 2$. Considering that we are complete for $a 1$ and $b 2$, as assumed above, we conclude that we are complete for all pupils selected by $Q_{\mathrm{sn}}$. Assume further that there exists a foreign key from pupil to class in our database, pupil [ccode, sname] $\subseteq$ class [ccode, sname]. Then we have all records of class that can be joined with pupil, i.e. we have all class records selected by $Q_{\mathrm{sn}}$. Overall, assuming the constraint and the foreign key, we can conclude that the answer to $Q_{\mathrm{sn}}$ is complete.

Related Work. Motro [17] was the first to formalize incomplete databases and completeness of queries. Levy [15], in addition, in- 
troduced a format for assertions that say which parts of a relational database are complete. We call these assertions table completeness or TC statements. He raised the problem to determine whether a set of such TC statements imply that some given query can be answered completely. Razniewski and Nutt [20] showed how to reduce this completeness reasoning problem to containment of conjunctive queries [4] and gave a comprehensive analysis of its complexity, considering several variants of queries and assertions. In particular, they showed that for the most expressive queries and assertions they considered, completeness reasoning is $\Pi_{2}^{P}$-complete. Such a degree of difficulty is reached, for instance, if queries and assertions are expressed by conjunctive queries and if finite-domain constraints hold over the database.

Contributions. In this work, we consider databases that are incomplete in that entire tuples are missing. We address the problem of completeness reasoning for conjunctive queries and for TC statements in the presence of constraints. We generalize the previously investigated problem by taking into consideration two kinds of constraints. The first are primary and foreign key constraints, the second one are a new kind of constraints, that we call conditional finite domain constraints (CFDCs), which restrict the domain of relation attributes depending on the values of other attributes. CFDCs are more expressive than classical finite domains constraints (FDCs). On the other hand, if we have a database instance at disposal, reasoning about completeness with a database instance is typically intractable [20], and therefore not feasible for large instances in realistic scenarios. In this respect, we expect that CFDCs offer a good trade-off that allows for feasible running times while still carrying more information than FDCs.

Further, we provide a new approach to completeness reasoning which gives rise to an implementation technique of the problem that was not obvious from previous work [19]. A demonstrator system, MAGIK, which realizes our approach and which is publicly accessible on the $\mathrm{Web}^{1}$ has been implemented based on the techniques in this paper and was presented in [21] and [22].

Our approach consists of two steps. First, we develop theorems that provide a syntactic characterization of the reasoning tasks for each combination of constraints. Based on these, we develop encodings of the problems into logic programming formalisms, where disjunction occurs in the head of the rules that encode CFDCs. One can run such encodings using Answer Set Programming (ASP) solvers (or Prolog engines if there are no CFDCs) such that the resulting program under cautious reasoning entails a test fact if and only if the original problem has the answer "yes". While logic programming is conceptually close to the characterizations, they can in principle also be used to establish encodings into other paradigms.

In the paper we present some of the proofs. For more technical ones we only provide the intuition, due to the space limitations. Complete proofs can be found in [18].

We tested our encoding using two state-of-the-art ASP solvers, $\mathrm{dlv}^{2}$ [14] and clingo ${ }^{3}$ [10], and one Prolog implementation, $\mathrm{Swi}^{4}$. The obtained results show that our encoding is able to process inputs that mirror the requirements of real world systems.

The remainder of the paper is organized as follows. In Section 2, we recall basic definitions from database theory and fix our notation. Section 3 formally introduces completeness reasoning. In Section 4 , we characterize completeness reasoning in the absence of constraints and present our encoding for this case. Characteri-

\footnotetext{
1 http://magik-demo.inf.unibz.it

2 http: //www.dlvsystem.com

3 http: //potassco.sourceforge.net

$4_{\text {http: / / www.swi-prolog.org }}$
}

zation and encoding are generalized in Section 5 to take account of key and foreign key constraints, and in Section 6 to conditional finite domain constraints into account, while the combination of both is studied in Section 7. Section 8 investigates the problem of finding a complete generalization of an incomplete query. Section 9 reports on our experiments and Section 10 concludes.

\section{PRELIMINARIES}

Relational Databases and Conjunctive Queries. We assume an infinite set of constants, dom, and a database schema, $\Sigma$, with an infinite set of relations. Each relation is identified by a relation name, e.g., $R$, and a list of attributes, $\operatorname{att}(R)$. We use $\operatorname{ary}(R)$ to denote the arity of $R$. In the following, we assume the schema to be fixed. For a relation $R$ with arity $n$, an atom is an expression $R\left(t_{1}, \ldots, t_{n}\right)$, where $t_{1} \ldots t_{n}$ are either elements of dom or variables. We denote constants with lower-case and variables with upper-case letters. A database instance $D$ is a finite set of ground atoms, that is, atoms that contain only constants. We sometimes refer to the atoms in an instance as facts. For a relation $R$, we denote as $R(D)$ the set $\{\bar{t} \mid R(\bar{t}) \in D\}$ of all tuples occurring in an $R$-atom in $D$. A condition is a set of atoms.

A conjunctive query is written as $Q(\bar{X}) \leftarrow B$, where $B$ is a condition and $\bar{X}$ is a tuple of variables, each of which occurs also in $B$. We call $B$ the body of $Q$, the variables in $\bar{X}$ the distinguished variables and the other variables in $B$ the nondistinguished variables. Given a conjunctive query $Q(\bar{X}) \leftarrow B$ and an instance $D$, an answer to $Q$ is a tuple $\alpha \bar{X}$, where $\alpha$ is an assignment of domain values to variables such that $\alpha B \subseteq D$. The set of all answers to $Q$ over $D$ is written as $Q^{s}(D)$. Similarly, we define $Q^{b}(D)$ as the bag of answers that contains as many copies of a tuple as there are assignments returning it. We say that $Q$ is evaluated under set or bag semantics, respectively, if we refer to the set or bag of answers.

Constraints. Database systems allow one to formulate conditions, so-called (integrity) constraints, that all instances of a database have to satisfy. Many of them can be captured in logic and allow for more inferences when reasoning about instances and queries. In this paper, we consider key constraints, foreign key constraints, and conditional finite domain constraints.

A primary key $(\mathrm{PK})$ constraint has the form $\operatorname{Key}(R, A)$, where $R$ is a relation symbol and $A$ is a sublist of $\operatorname{att}(R)$. A foreign key (FK) constraint has the form $R[A] \subseteq S[B]$, where $R, S$, are relations, $A$ is a sublist of $\operatorname{att}(R)$, and $B$ is from $\operatorname{Key}(S, B)$. Satisfaction of PK and FK constraints are defined as usual (cf. [1]).

In this paper, we also consider weakly acyclic sets of FK constraints, which have been introduced by Fagin et al. For the definition, we refer to their paper [7].

A conditional finite domain (CFD) constraint has the form $R\{A=$ $\bar{v}\}[a]=W$, where $R$ is a relation, $A$ is a sublist of $\operatorname{att}(R), \bar{v}$ is a list of constants of the same length as $A, a$ is an attribute from $\operatorname{att}(R)$ that is not in $A$, and $W$ is a set of constants. An instance $D$ satisfies such a constraint if for every fact $R(\bar{t}) \in D$ we have that if $R(\bar{t})[A]=\bar{v}$ then $R(\bar{t})[a] \in W$, where $R(\bar{t})[A]$ is the projection of the values of $R(\bar{t})$ at the attribute places from $A$ and $R(\bar{t})[a] \in W$ is defined similarly. Alternatively, if $A=a_{1}, \ldots, a_{n}$ and $\bar{v}=v_{1}, \ldots, v_{n}$, we write the condition $A=\bar{v}$ as a list of equalities, $a_{1}=v_{1}, \ldots, a_{n}=v_{n}$. For example, the CFD constraint that states "a class at Newton school from the science branch has as code either $a 1$ or $b 2$ ", is expressed as class $\{$ school $=$ newton, branch $=s c i\}[$ ccode $]=\{a 1, b 2\}$. If $A$ and $\bar{v}$ are empty in a CFD constraint, we simply write $R[a]=W$ and call this a finite domain (FD) constraint.

We will use $\mathcal{K}$ and $\mathcal{F}$ generically to denote sets of FK, and CFD constraints, respectively. 
Logic Programming. A disjunctive rule has the form

$$
A_{1}|\ldots| A_{k} \leftarrow B_{1}, \ldots, B_{n},
$$

where the $A$ 's and $B$ 's are atoms (see [11]). If $k=1$, that is, the head consists of a single atom, we say that the rule is a Horn rule. A fact is a Horn rule with empty body (we omit " $\leftarrow$ ”). A rule with empty head (the empty disjunction) is a denial. (We avoid the common term "integrity constraint" in this paper to avoid confusion with integrity constraints over databases.) An answer-set program is a finite set of disjunctive rules. For the theory of answer set programs we refer to [23] and [3]. A positive logic program contains only Horn rules. Positive logic programs can be executed under SLD resolution as implemented in Prolog [2].

\section{DATA COMPLETENESS}

Running Example. Our examples build upon a toy schema from the school world with the three relations

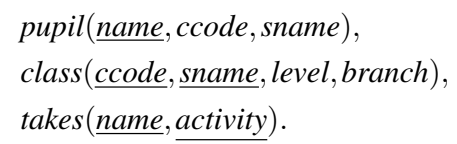

Here, pupil(fred,al,newton) means that Fred is a pupil of class al at Newton school; class(al,newton, 1, sci) means that class al at Newton school is a $1^{\text {st }}$ level class that belongs to the science branch; and takes (fred,chess) means that Fred is taking chess as an extracurricular activity.

Underlined attributes indicate the primary key, which in our notation can be expressed as the key constraints $\operatorname{Key}($ pupil,$\{$ name $\})$, $\operatorname{Key}($ class,$\{$ ccode, sname $\})$, and Key(takes, $\{$ name, activity $\})$. We also consider two FK constraints, which say that every class, identified by class code and school name, referred to by a pupil tuple occurs in the class relation, and every pupil name referred to by a takes tuple occurs in the pupil relation. Formally, this is expressed as pupil[ccode, sname $] \subseteq$ class $[$ ccode, sname $]$ and takes $[$ name $] \subseteq$ pupil[name]. Finally, we consider a CFD constraint that restricts classes at Newton school to belong either to the science or the humanities branch, expressed as class $\{$ sname $=$ newton $\}[$ branch $]=$ $\{$ sci,hum $\}$.

The conjunctive query $Q_{\mathrm{s} 1 \mathrm{n}}$, defined by the rule

$$
Q_{\mathrm{s} 1 \mathrm{n}}(N) \leftarrow \operatorname{pupil}(N, C, \text { newton }), \operatorname{class}(C, \text { newton, } 1, \text { sci })
$$

asks for "the names of all pupils from Newton school of the $1^{\text {st }}$ level that attend a class from the science branch".

Query and Table Completeness. When stating that data is incomplete, one must have a conceptual complete reference. We model an incomplete database in the style of [17] as a pair of database instances $\mathcal{D}=\left(D^{i}, D^{a}\right)$, where $D^{a} \subseteq D^{i}$. Here, $D^{i}$ is called the ideal state and $D^{a}$ the available state. In an application, the state stored in a DBMS is the available state, which often represents only a part of the facts that hold in reality. The facts holding in reality constitute the ideal state, which however is unknown. (Later on we will introduce table completeness statements as a way to express meta-information about the extent to which the available state captures the ideal state.)

Data are accessed by posing queries. We would like to know whether a database has sufficient information to answer a query completely, that is, whether the query is complete. If we can infer from meta-information that a query is complete, we know that the answer we receive over the available database is the same as the one we would get over the (hypothetical) ideal database.
We write $\operatorname{Compl}^{s}(Q)$ and $\operatorname{Compl}^{b}(Q)$, respectively, to indicate that $Q$ is complete under set or bag semantics. The first statement is satisfied by an incomplete database $\mathcal{D}=\left(D^{i}, D^{a}\right)$, written $\mathcal{D} \models \operatorname{Compl}^{s}(Q)$, if $Q^{s}\left(D^{i}\right)=Q^{s}\left(D^{a}\right)$. Analogously, the second is satisfied if $Q^{b}\left(D^{i}\right)=Q^{b}\left(D^{a}\right)$.

With table completeness (TC) statements we specify that parts of a table are complete. A TC statement, written $\operatorname{Compl}(R(\bar{s}) ; G)$, has two components, a relational atom $R(\bar{s})$ and a condition $G$. In the sequel, we will denote a TC statement generically as $C$. As an example, consider

$$
\begin{aligned}
C_{\mathrm{sci}} & =\operatorname{Compl}(\operatorname{pupil}(N, C, S) ; \operatorname{class}(C, S, L, \operatorname{sci})) \\
C_{\mathrm{lev} 1} & =\operatorname{Compl}(\operatorname{class}(C, S, 1, B) ; \text { true }) .
\end{aligned}
$$

The first statement asserts that the table pupil contains all records of pupils from science classes, while the second asserts that the table class contains all records of classes at level 1.

To define the semantics of a TC statement $C$, we associate a query $Q_{C}(\bar{s}) \leftarrow R(\bar{s}), G$ to it. Then $C$ is satisfied by $\mathcal{D}=\left(D^{i}, D^{a}\right)$, written $\mathcal{D}=\operatorname{Compl}(R(\bar{s}) ; G)$, if $Q_{C}\left(D^{i}\right) \subseteq R\left(D^{a}\right)$. This means that the ideal instance $D^{i}$ is used to determine those tuples in the ideal version $R\left(D^{i}\right)$ that satisfy $G$, and that the statement $C$ is satisfied if these tuples are present in the available version $R\left(D^{a}\right)$. We refer to the query associated to $C$ as $Q_{C}$. For instance, the query associated to $C_{\mathrm{sci}}$ is $Q_{C_{\mathrm{sci}}}(N, C, S) \leftarrow \operatorname{pupil}(N, C, S), \operatorname{class}(C, S, L, s c i)$.

To reason about completeness, we define for every set $\mathcal{C}$ of TC statements the operator $T_{\mathcal{C}}$ that maps database instances to database instances. If $C$ is a TC statement about $R$, then we define $T_{C}(D):=$ $\left\{R(\bar{t}) \mid \bar{t} \in Q_{C}(D)\right\}$. For $\mathcal{C}$ we define

$$
T_{\mathcal{C}}(D):=\bigcup_{C \in \mathcal{C}} T_{C}(D) .
$$

The operator $T_{\mathcal{C}}$ is monotonic, and for every instance $D$, the pair $\left(D, T_{\mathcal{C}}(D)\right)$ is an incomplete database satisfying $\mathcal{C}$, and $T_{\mathcal{C}}(D)$ is the smallest set (wrt set inclusion) for which this holds. We formalize this with the following claim that we need for the proofs later on.

Proposition 1 ([20]). Let $\mathcal{C}$ be a set of TC statements. Then

a) $T_{\mathcal{C}}(D) \subseteq D, \quad$ for all database instances $D$;

b) $\left(D^{i}, D^{a}\right) \models \mathcal{C}$ iff $T_{\mathcal{C}}\left(D^{i}\right) \subseteq D^{a}$, for all $D^{a} \subseteq D^{i}$.

We say that $\mathcal{C}$ entails the completeness of $Q$ with respect to set semantics, written $\mathcal{C} \models \operatorname{Compl}^{s}(Q)$, if every incomplete database $\mathcal{D}$ that satisfies the statements in $\mathcal{C}$, also satisfies $\operatorname{Compl}^{s}(Q)$. The meaning of "C $\models \operatorname{Compl}^{b}(Q)$ " is defined analogously.

The completeness reasoning problem is to check, given $\mathcal{C}$ and $Q$, whether the entailments above hold. An instance of this problem for set semantics is to check whether $\left\{C_{\text {sci }}, C_{\text {lev1 } 1}\right\} \mid=\operatorname{Compl}^{S}\left(Q_{\text {s1n }}\right)$ (which intuitively holds and which we prove to hold in Section 4).

Let $\mathcal{F}$ be a set of CFD constraints and $\mathcal{K}$ be a set of key and FK constraints. We say that $\mathcal{D}=\left(D^{i}, D^{a}\right)$ satisfies $\mathcal{F}$ (resp., $\mathcal{K}$ ) if $D^{i}$ satisfies $\mathcal{F}$ (resp., $\mathcal{K}$ ). Note that, due to $D^{a} \subseteq D^{i}$, this implies that also $D^{a}$ satisfies $\mathcal{F}$ and that $D^{a}$ satisfies the key constraints in $\mathcal{K}$. We say that $\mathcal{K}$ is enforced over $\mathcal{D}$ if both $D^{i}$ and $D^{a}$ satisfy $\mathcal{K}$. If $\mathcal{D}$ satisfies $\mathcal{K}$, this means that in the real world, represented by the ideal database, the $\mathrm{FK}$ constraints hold. If $\mathcal{K}$ is enforced over $\mathcal{D}$, then the constraints of $\mathcal{K}$ hold also over the available database. For instance, we may know that all pupils at our school belong to a class (because there are no external pupils), but the information about the levels and branches of classes contained in the relation class has not been entered into the available database. In that case, $\mathcal{D}$ satisfies pupil $[$ ccode, sname $] \subseteq$ class $[$ ccode, sname $]$, but the constraint is not enforced. 
We say that $\mathcal{C}$ entails the completeness of $Q$ wrt $\mathcal{F}$, and write $\mathcal{C}={ }_{\mathcal{F}} \operatorname{Compl}^{s}(Q)$, if every $\mathcal{D}$ that satisfies both $\mathcal{C}$ and $\mathcal{F}$, also satisfies $\operatorname{Compl}^{s}(Q)$. Analogously, we define " $=\mathcal{K}$ ", while " $={ }_{\mathcal{K}}^{e}$ " means that only such $\mathcal{D}$ 's are considered where $\mathcal{K}$ is enforced.

From [20] we know that the completeness reasoning problem, both for set and bag semantics, can be reduced to query containment (cf. [4] and [13]). Reasoning in the presence of FD constraints can be reduced to containment with respect to FD constraints [19].

\section{PLAIN COMPLETENESS REASONING}

In previous work, Razniewski and Nutt reduced completeness reasoning to query containment by which they readily obtained complexity results [20]. However, the reduction did not give rise to an implementation technique. In this paper, we will reduce completeness checking to the question whether a test query returns a specific result over a test database. We will then use these characterizations to translate completeness checks into logic programs that can be executed by ASP or Prolog engines.

In the rest of the paper, we always consider a set of TC statements $\mathcal{C}$ and a conjunctive query $Q$ defined by the rule $Q(\bar{X}) \leftarrow$ $R_{1}\left(\bar{t}_{1}\right), \ldots, R_{n}\left(\bar{t}_{n}\right)$.

Characterizing Completeness Reasoning. While developing our approach, we illustrate it with an example. Consider the query $Q_{\text {s1n }}$ in (1) and the set $\mathcal{C}_{\text {sci,lev } 1}=\left\{C_{\text {sci }}, C_{\text {lev } 1}\right\}$ comprising the TC statements in (2) and (3). Suppose $\mathcal{D}=\left(D^{i}, D^{a}\right)$ satisfies $\mathcal{C}_{\text {sci,lev1 }}$. Consider an answer, say $n^{\prime}$, returned by $Q_{\text {sin }}$ over the ideal instance $D^{i}$. Then $D^{i}$ contains two atoms of the form $\operatorname{pupil}\left(n^{\prime}, c^{\prime}\right.$, newton $)$ and $\operatorname{class}\left(c^{\prime}\right.$, newton, 1, sci $)$. Now, due to $C_{\mathrm{sci}}$, also $D^{a}$ contains $\operatorname{pupil}\left(n^{\prime}, c^{\prime}\right.$, newton $)$, and due to $C_{\mathrm{lev} 1}$, the atom $\operatorname{class}\left(c^{\prime}\right.$, newton, 1 , $s c i$ ) is in $D^{a}$, too. Consequently, $Q_{\text {sin }}$ returns $n^{\prime}$ also over $D^{a}$. Since $D^{i}$ and $D^{a}$ were arbitrary, this shows that $\mathcal{C}_{\text {sci,lev1 }} \models \operatorname{Compl}^{S}\left(Q_{\text {s1n }}\right)$.

Using the $T_{\mathcal{C}}$ transformation in (4), we can generalize this approach to a completeness test. We define the set of facts $D_{Q}$, which we call the canonical database of $Q$, obtained by freezing the atoms in the body of $Q$. ("Freezing" variables is a well-known concept in logic programming and database theory, which allows one to treat a variable like a constant.) Thus,

$$
D_{Q}=\left\{R_{1}\left(\theta \bar{t}_{1}\right), \ldots, R_{n}\left(\theta \bar{t}_{n}\right)\right\},
$$

where $\theta$ is the substitution that maps each variable $X$ to the "frozen version" $\theta X$ of $X$.

To check whether $Q$ is complete we apply $T_{\mathcal{C}}$ to $D_{Q}$ and check whether $Q$ can retrieve the frozen tuple of distinguished variables.

Theorem 2 (Characterization Set Semantics). Let $\mathcal{C}$ be a set of TC statements, and $Q(\bar{X}) \leftarrow B$ be a conjunctive query. Then

$$
\mathcal{C} \models \operatorname{Compl}^{s}(Q) \quad \Longleftrightarrow \quad \theta \bar{X} \in Q\left(T_{\mathcal{C}}\left(D_{Q}\right)\right) .
$$

Proof. $(\Rightarrow)$ Suppose that $\mathcal{C} \models \operatorname{Compl}^{s}(Q)$. To show that $\theta \bar{X} \in$ $Q\left(T_{\mathcal{C}}\left(D_{Q}\right)\right)$, we construct the pair $\mathcal{D}_{Q}=\left(D_{Q}, T_{\mathcal{C}}\left(D_{Q}\right)\right)$, which is an incomplete database according to Proposition $1 \mathrm{a})$. Obviously, it holds that $T_{\mathcal{C}}\left(D_{Q}\right) \subseteq T_{\mathcal{C}}\left(D_{Q}\right)$, and thus from Proposition $1 \mathrm{~b}$ ) we conclude that $\mathcal{D}_{Q} \mid \mathcal{C}$. Hence, $\mathcal{D}_{Q}=\operatorname{Compl}^{s}(Q)$ and $Q\left(D_{Q}\right)=$ $Q\left(T_{\mathcal{C}}\left(D_{Q}\right)\right)$. Further, $D_{Q}$ is the frozen version of the body of $Q$, where the output variables $\bar{X}$ have been substituted with $\theta \bar{X}$. Thus $\theta \bar{X} \in Q\left(D_{Q}\right)$, and it follows that $\theta \bar{X} \in Q\left(T_{\mathcal{C}}\left(D_{Q}\right)\right)$.

$(\Leftarrow)$ Suppose that $\theta \bar{X} \in Q\left(T_{\mathcal{C}}\left(D_{Q}\right)\right)$. We want to show that $\mathcal{C}=\operatorname{Compl}^{s}(Q)$. To this end let $\mathcal{D}=\left(D^{i}, D^{a}\right)$ be an incomplete database such that $\mathcal{D}=\mathcal{C}$. Suppose that $\bar{c} \in Q\left(D^{i}\right)$. We show that $\bar{c} \in Q\left(D^{a}\right)$. Let $\alpha$ be an assignment satisfying $Q$ over $D^{i}$ that retrieves $\bar{c}$. Then $\alpha \bar{X}=\bar{c}$ and $\alpha B \subseteq D^{i}$, where $B$ is the body of $Q$.
Since $\mathcal{C} \models \operatorname{Compl}^{s}(Q)$, it follows that $T_{\mathcal{C}}(\alpha B) \subseteq D^{a}$. Now, let $\beta$ be a satisfying assignment for $Q$ over $T_{\mathcal{C}}\left(D_{Q}\right)$ that retrieves $\theta \bar{X}$. Then $\beta \bar{X}=\theta \bar{X}$ and $\beta B \subseteq T_{\mathcal{C}}\left(D_{Q}\right)=T_{\mathcal{C}}(\theta B)$. Consider the composed assignment $\alpha \theta^{-1} \beta$. Because of the inclusion just derived, it holds that $\alpha \theta^{-1} \beta B \subseteq \alpha \theta^{-1} T_{\mathcal{C}}(\theta B)$. It also holds that $\alpha \theta^{-1} T_{\mathcal{C}}(\theta B) \subseteq$ $T_{\mathcal{C}}\left(\alpha \theta^{-1} \theta B\right)$, since $\alpha$ may map a variable to a constant appearing in $\mathcal{C}$, or may map distinct variables to the same constant, thus enabling more TC statements in $\mathcal{C}$ to be applied. Moreover, we have that $T_{\mathcal{C}}\left(\alpha \theta^{-1} \theta B\right)=T_{\mathcal{C}}(\alpha B) \subseteq D^{a}$, where the last inclusion has been derived above. In summary, we have shown that $\alpha \theta^{-1} \beta$ satisfies $Q$ over $D^{a}$. We verify that $\bar{c}=\alpha \bar{X}=\alpha \theta^{-1} \theta \bar{X}=\alpha \theta^{-1} \beta \bar{X}$, which implies that $\bar{c}$ is the result retrieved by $Q$ over $D^{a}$ with the assignment $\alpha \theta^{-1} \beta$, so that $\bar{c} \in Q\left(D^{a}\right)$.

Encoding Completeness Reasoning. For any $Q$ and $\mathcal{C}$, the check whether $\theta \bar{X} \in Q\left(T_{\mathcal{C}}\left(D_{Q}\right)\right)$ can be encoded into a positive logic program. As a set of facts, the program contains the atoms in $D_{Q}$. Then we extend our signature by two additional relation symbols $R^{i}$ and $R^{a}$ for every $R$ in $\Sigma$, to be able to reason about the ideal and available instances. We also introduce a copy rule $r_{R}: R^{i}(\bar{X}) \leftarrow R(\bar{X})$ for every relation and denote the set of all such copy rules as $P_{\Sigma}$. Thus, any model of $D_{Q}$ and $P_{\Sigma}$ contains an "ideal" copy of $D_{Q}$.

Next, to capture the reasoning with TC statements, we introduce for each $C \in \mathcal{C}, C=\operatorname{Compl}(R(\bar{s}) ; G)$, the rule $r_{C}$, defined as:

$$
R^{a}(\bar{s}) \leftarrow R^{i}(\bar{s}), G^{i},
$$

where the $i$ in $G^{i}$ indicates that all relation symbols are replaced by their ideal version. For example, $r_{C_{\text {sci }}}$ is the rule pupil ${ }^{a}(N, C, S) \leftarrow$ $\operatorname{pupil}^{i}(N, C, S), \operatorname{class}^{i}(C, S, L, s c i)$ and $r_{C_{\mathrm{lev}}}$ the rule $\operatorname{class}^{a}(C, S, 1$, $B) \leftarrow \operatorname{class}^{i}(C, S, 1, B)$. The set of all rules for statements in $\mathcal{C}$ is

$$
P_{\mathcal{C}}=\left\{r_{C} \mid C \in \mathcal{C}\right\} .
$$

Intuitively, $D_{Q}$ is a prototypical instance where $Q$ returns an answer, namely, the prototypical answer $\theta \bar{X}$. Applying the copy rules in $P_{\Sigma}$ turns $D_{Q}$ into an ideal database $D_{Q}^{i}$. In the following, we use ideal $(D)$ or $D^{i}$ (resp., avail $(D)$ or $D^{a}$ ) to denote the ideal copy (resp., the available copy) of the instance $D$. The application of the statement rules in $P_{\mathcal{C}}$ then amounts to computing the available instance avail $\left(T_{\mathcal{C}}\left(D_{Q}^{i}\right)\right)$. It remains to check whether $Q$ returns the answer $\theta \bar{X}$ also over avail $\left(T_{\mathcal{C}}\left(D_{Q}^{i}\right)\right)$.

To this end, we introduce the boolean test query $Q^{s}$, which is obtained from $Q$ by replacing each relation symbol by its available version and freezing the distinguished variables. Formally, $Q^{s}$ is defined by the rule $r_{Q^{s}}=Q^{s} \leftarrow R_{1}^{a}\left(\theta \bar{t}_{1}\right), \ldots, R_{n}^{a}\left(\theta \bar{t}_{n}\right)$, where $\theta$ is the substitution that maps every distinguished variable to its frozen version. In our example, the test query is $Q_{\mathrm{s} 1 \mathrm{n}}^{s} \leftarrow$ pupil ${ }^{a}\left(n^{\prime}\right.$, $C$, newton $),$ class $^{a}(C$, newton, 1, sci $)$.

In addition to the program $P_{\mathcal{C}}$, encoding $\mathcal{C}$, we define $P_{Q}^{S}:=D_{Q} \cup$ $P_{\Sigma} \cup\left\{r_{Q^{s}}\right\}$, as the program encoding $Q$.

THEOREM 3 (SET ENCODING). Let $Q$ be a conjunctive query and $\mathcal{C}$ be a set of TC statements. Then $\mathcal{C} \models \operatorname{Compl}^{S}(Q)$ iff

1. the atom $Q^{s}$ is in the answer set of $P_{\mathcal{C}} \cup P_{Q}^{S}$, or

2. the goal $Q^{s}$ succeeds with $P_{\mathcal{C}} \cup P_{Q}^{s}$ under $S L D$ resolution.

Proof. 1. To prove the claim it is sufficient to show that $\theta \bar{X} \in$ $Q\left(T_{\mathcal{C}}\left(D_{Q}\right)\right)$ if and only if the atom $Q^{s}$ is in the answer set of $P_{\mathcal{C}} \cup$ $P_{Q}^{s}$. From this and Theorem 2 the claim follows directly. Since $P_{\mathcal{C}} \cup P_{Q}^{S}$ is a positive program, it has a unique answer set that corresponds to the least fixed point (LFP) of the program. By the program definition, the LFP of the program is consists of the facts in 
(i) $D_{Q}$, (ii) the ideal copy $D_{Q}^{i}$ produced by $\mathcal{P}_{\Sigma}$, (iii) the available version avail $\left(T_{\mathcal{C}}\left(D_{Q}\right)\right)$ of $T_{\mathcal{C}}\left(D_{Q}\right)$, obtained by applying the program $\mathcal{P}_{\mathcal{C}}$ on $D_{Q}^{i}$, and (iv) possibly the ground atom $Q_{s}$ that can be produced by the rule $r_{Q^{s}}$ over the facts from avail $\left(T_{\mathcal{C}}\left(D_{Q}\right)\right)$. Thus, we have that $Q^{s}$ is in the LFP of the encoding program if and only if $\theta \bar{X} \in Q\left(T_{\mathcal{C}}\left(D_{Q}\right)\right)$.

2. For SLD resolution it holds that each SLD-provable ground atom of a program belongs to the least Herbrand model (LHM) of the program. Since, in our case, the LFP of the answer set program is the same as the LHM of the Prolog program, the claim follows from Case 1.

Recall that in the definition of the test query, we freeze the distinguished variables because we want to test whether $Q$ returns $\theta \bar{X}$, and we do not freeze the non-distinguished variables because we do not want to impose constraints on how $\theta \bar{X}$ is retrieved. To see why this definition works, consider the query

$$
Q_{\text {cht }}(N) \leftarrow \operatorname{takes}(N, \text { chess }) \text {, takes }(N, A),
$$

which asks for "all pupils that take chess, which in addition take some activity" (which may be chess). Suppose, our data are complete for all pupils that take chess, expressed by the TC statement

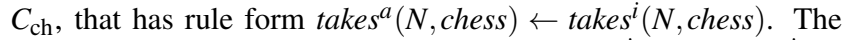
ideal copy of the canonical database $D_{Q_{\text {cht }}}$ is $D_{Q_{\text {cht }}}^{i}=\left\{\right.$ takes $^{i}\left(n^{\prime}\right.$, chess), $\left.\operatorname{takes}^{i}\left(n^{\prime}, a^{\prime}\right)\right\}$. With our TC rule, we can derive the fact takes $^{a}\left(n^{\prime}\right.$, chess $)$, but not takes ${ }^{a}\left(n^{\prime}, a^{\prime}\right)$. Still, this is enough to suc-

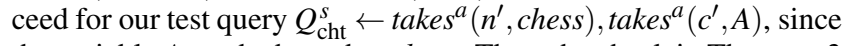
the variable $A$ can be bound to chess. Thus, the check in Theorem 3 returns the intuitively expected answer "yes".

Completeness Under Bag Semantics. Under bag semantics, a query returns an answer tuple as many times as there are satisfying assignments for that tuple. Thus, a set $\mathcal{C}$ may entail completeness of $Q$ under set, but not under bag semantics. The query $Q_{\text {cht }}$ in (5) returns each pupil that takes chess as many times as there are activities taken by that pupil. Thus, under bag semantics the additional atom $\operatorname{takes}(N, A)$ makes sense, as it does not change the answers as such, but the multiplicity with which they appear. The query now asks, "How many activities does each chess taker take?"

For reasoning under bag semantics, it no longer suffices to check whether each answer of $Q$ over $D^{i}$ is also an answer over $D^{a}$, but whether each satisfying assignment for $Q$ over $D^{i}$ is retained over $D^{a}$. Intuitively, the freezing assignment $\theta$ is a prototypical satisfying assignment for $Q$ over the prototypical ideal database $D_{Q}$. The minimal available database that, together with $D_{Q}$, satisfies $\mathcal{C}$, is $T_{\mathcal{C}}\left(D_{Q}\right)$. To test whether $\theta$ is also a satisfying assignment over $T_{\mathcal{C}}\left(D_{Q}\right)$, we simply check whether $D_{Q} \subseteq T_{\mathcal{C}}\left(D_{Q}\right)$.

Theorem 4 (Characterization Bag SEMAntics). Let $\mathcal{C}$ be a set of TC statements, and $Q$ be a conjunctive query. Then

$$
\mathcal{C}=\operatorname{Compl}^{b}(Q) \quad \Longleftrightarrow \quad D_{Q} \subseteq T_{\mathcal{C}}\left(D_{Q}\right) .
$$

PROOF. $(\Rightarrow)$ As in the proof of Theorem 2, we observe that $\left(D_{Q}, T_{\mathcal{C}}\left(D_{Q}\right)\right) \models \mathcal{C}$. Hence, $\left(D_{Q}, T_{\mathcal{C}}\left(D_{Q}\right)\right) \models \operatorname{Compl}^{b}(Q)$, that is, the number of times a tuple occurs as an answer of $Q$ over $T_{\mathcal{C}}\left(D_{Q}\right)$ is the same it occurs over $D_{Q}$. Since $T_{\mathcal{C}}\left(D_{Q}\right) \subseteq D_{Q}$, this is only possible if every assignment that satisfies $Q$ over $D_{Q}$ also satisfies $Q$ over $T_{\mathcal{C}}\left(D_{Q}\right)$. In particular, the freezing mapping $\theta$ is an assignment that satisfies $Q$ over $D_{Q}=\theta B$ where $B$ is the body of $Q$. By the argument above, $\theta$ also satisfies $Q$ over $T_{\mathcal{C}}\left(D_{Q}\right)$, and hence $\theta B \subseteq T_{\mathcal{C}}\left(D_{Q}\right)$, that is, $D_{Q} \subseteq T_{\mathcal{C}}\left(D_{Q}\right)$.

$(\Leftarrow)$ Suppose $D_{Q} \subseteq T_{\mathcal{C}}\left(D_{Q}\right)$. Let $\mathcal{D}=\left(D^{i}, D^{a}\right) \models \mathcal{C}$ for an incomplete database $\mathcal{D}$ and let $\bar{c} \in Q\left(D^{i}\right)$. We show that $\bar{c}$ appears the same number of times as answer to $Q$ over $D^{a}$ as it does over $D^{i}$. Since $D^{a} \subseteq D^{i}$, it suffices to show that each assignment $\alpha$ returning $\bar{c}$ over $D^{i}$ also returns $\bar{c}$ over $D^{a}$. Similar to the proof for the case of set semantics, one can show that $T_{\mathcal{C}}(\alpha B) \subseteq D^{a}$. Further, from $D_{Q} \subseteq T_{\mathcal{C}}\left(D_{Q}\right)$ it follows that $\alpha \theta^{-1}(\theta B) \subseteq \alpha \theta^{-1} T_{\mathcal{C}}(\theta B)$. Next, $\alpha \theta^{-1} T_{\mathcal{C}}(\theta B) \subseteq T_{\mathcal{C}}\left(\alpha \theta^{-1} \theta B\right)=T_{\mathcal{C}}(\alpha B)$ for the same reasons as in the proof of Theorem 2. Altogether, $\alpha B \subseteq T_{\mathcal{C}}(\alpha B)$. Then, from $\alpha B \subseteq T_{\mathcal{C}}(\alpha B) \subseteq D^{a}$ we conclude $\alpha B \subseteq D^{a}$. This implies that $\alpha$ is also a satisfying assignment for $Q$ over $D^{a}$, which returns $\bar{c}$.

This characterization can be readily encoded into a logic program. Instead of freezing only the distinguished variables of $Q$, we freeze all variables in $Q$. We thus define $Q^{b}$ by the rule $r_{Q^{b}}=$ $Q^{b} \leftarrow R_{1}^{a}\left(\theta \bar{t}_{1}\right), \ldots, R_{n}^{a}\left(\theta \bar{t}_{n}\right)$, where now $\theta$ is the substitution that maps every variable to its frozen version. The encoding of $Q$ is now $P_{Q}^{b}:=D_{Q} \cup P_{\Sigma} \cup\left\{r_{Q^{b}}\right\}$.

TheOREM 5 (BAG ENCODING). Let $Q$ be a conjunctive query and $\mathcal{C}$ be a set of TC statements. Then $\mathcal{C}=\operatorname{Compl}^{b}(Q)$ iff

1. the atom $Q^{b}$ is in the answer set of $P_{\mathcal{C}} \cup P_{Q}^{b}$, or

2. the goal $Q^{b}$ succeeds with $P_{\mathcal{C}} \cup P_{Q}^{b}$ under $S L D$ resolution.

Proof SKETCH. 1. Due to Theorem 4, it suffices to prove that the atom $Q^{b}$ is in the answer set of $P_{\mathcal{C}} \cup P_{Q}^{b}$ iff $D_{Q} \subseteq T_{\mathcal{C}}\left(D_{Q}\right)$. Similarly to the case of set semantics, this can be proved by showing that the test $D_{Q} \subseteq T_{\mathcal{C}}\left(D_{Q}\right)$ succeeds if and only if the rule $r_{Q^{b}}$ fires.

2. As for Theorem 3, the claim follows from the fact that a ground atom can be SLD-resolved iff it belongs to the least Herbrand model (LHM) of the program. In our encoding, the LHM corresponds to least fixed point of the program. Thus, the claim follows from Claim 1.

The tests in Theorems 2 and 4 can be performed in nondeterministic polynomial time, which complies with the overall complexity of the problems, which are NP-complete.

\section{REASONING UNDER FOREIGN KEYS}

Foreign keys can help us draw additional conclusions about query completeness. We provide an example for both the effects of unenforced and enforced FKs.

Example. Consider the query $Q_{\mathrm{n}}(N) \leftarrow \operatorname{pupil}(N, C$, newton $)$. Recall that for each class, identified by its code and the name of its school, the relation class records the level and branch of that class. Assume that our database is "complete for those pupils at Newton school that attend a class that belongs to some level and branch," expressed as Compl(pupil( $N, C$, newton); $\operatorname{class}(C$, newton, $L, B))$. If there may exist classes at Newton school present in the pupil relation but not in class relation, then the completeness of $Q_{\mathrm{n}}$ does not follow. However, if we know that every class in the application domain has a level and belongs to a branch, we can express this by stating that the FK pupil [ccode, sname] $\subseteq$ class [ccode, sname] holds (without being enforced). In this case, for every pupil record there exists a corresponding class record in the ideal database, hence the above TC statement is enough to guarantee the completeness of $Q_{\mathrm{n}}$.

However, if we ask for all pupils attending a class of the science branch at Newton school, $Q_{\mathrm{sn}}(N) \leftarrow \operatorname{pupil}(N, C$, newton $), \operatorname{class}(C$, newton, $L, s c i)$, then the non-enforced FK does not guarantee completeness, since then for every pupil record a corresponding class record exists in the ideal database, representing the state of the real world, but such class records may not be present in the available database so that we cannot identify classes of the science branch. If 
the FK holds and is enforced, then all such records must be present, and the completeness of $Q_{\text {sn }}$ is ensured.

We will characterize query completeness in the presence of key and weakly acyclic FK constraints, both for non-enforced and enforced semantics. We assume that the bodies of the conjunctive queries to be checked for completeness satisfy the key constraints, that is, if $A$ is the list of key attributes of $R$ and if $R(\bar{s})$ and $R(\bar{t})$ are two atoms in $R$ such that $R(\bar{s})[A]=R(\bar{t})[A]$, then $\bar{s}=\bar{t}$. Satisfaction of key constraints can be achieved efficiently by chasing the body with the key constraints (see [12]).

The conceptual tool needed for foreign key reasoning is the chase with inclusion dependencies (IDs), which generalize foreign-key constraints. Johnson and Klug defined two versions, the oblivious chase, which introduces a new atom whenever an ID is "applicable," and the restricted or standard chase, which only introduces a new atom for an applicable ID if the ID is not yet satisfied [12]. We will use an intermediate chase version, introduced by Marnette [16], the oblivious Skolem chase, which generates atoms with Skolem terms whenever an ID is applicable, and the atom to be generated does not exist yet. The oblivious Skolem chase bears some similarity with the restricted chase in that a chase step is blocked if the corresponding ID is satisfied by an atom with Skolem terms.

To apply this chase, IDs are translated into rules with Skolem terms in the head. For instance, the foreign key from pupil to class is translated into the rule

$$
\begin{aligned}
\operatorname{class}\left(C, S, f_{\text {class }, \text { level }}(C, S), f_{\text {class, branch }}(C, S)\right) & \leftarrow \\
& \operatorname{pupil}(N, C, S),
\end{aligned}
$$

which introduces a new class atom for every pupil atom, with a new level and branch generated by two Skolem functions that take the keys of the class atom as arguments. Let $\mathcal{K}$ be a set of FKs. We denote the result of chasing a set of (ground) atoms $D$ with the rules obtained from $\mathcal{K}$ as $C h_{\mathcal{K}}(D)$. If $\mathcal{K}$ is clear from the context, we drop the subscript and write $C h(D)$. In general, chase termination is not guaranteed for FKs.

From now on, we consider only weakly acyclic sets of FKs [7]. Weakly acyclic tuple generating dependencies (which are more general constraints than FKs) have been introduced in the context of data exchange as syntactic class that ensures termination of the restricted chase for every instance [7]. Marnette extended this result to the oblivious Skolem chase [16]. Under this assumption, $C h_{\mathcal{K}}(D)$ is always finite for a finite $D$.

Now, we can formulate a result for non-enforced FKs that generalizes Theorems 2 and 4 by replacing the prototypical database $D_{Q}$ with the chase of $D_{Q}$.

THEOREM 6 (CHARACTERIZATION NON-ENFORCED FKS). Let $\mathcal{C}$ be a set of TC statements, $\mathcal{K}$ be a weakly acyclic set of $F K s$, and $Q$ be a conjunctive query. Then

$$
\begin{aligned}
& \text { 1. } \mathcal{C} \models_{\mathcal{K}} \operatorname{Compl}^{s}(Q) \quad \Longleftrightarrow \theta \bar{X} \in Q\left(T_{\mathcal{C}}\left(C h_{\mathcal{K}}\left(D_{Q}\right)\right)\right) \\
& \text { 2. } \mathcal{C} \models_{\mathcal{K}} \operatorname{Compl}^{b}(Q) \quad \Longleftrightarrow \quad D_{Q} \subseteq T_{\mathcal{C}}\left(C h_{\mathcal{K}}\left(D_{Q}\right)\right) .
\end{aligned}
$$

PROOF IDEA. 1. $(\Rightarrow)$ The proof is based on a similar construction as the one for Theorem 2. In this case we take $\mathcal{D}_{Q}=\left(C h\left(D_{Q}\right)\right.$, $\left.T_{\mathcal{C}}\left(C h\left(D_{Q}\right)\right)\right)$ as an incomplete database.

$(\Leftarrow)$ This direction is more involved. We have to cope with the difficulty that applying our chase to a database instance that satisfies the key constraints in $\mathcal{K}$ may result in an instance that no more satisfies the keys because of additional atoms with Skolem terms. For instance, chasing \{pupil(fred, a1, newton), class(a1,newton, 1 , $s c i)\}$ with rule (6) adds the atom class(a1, newton, $f_{\text {class,level }}(a 1$, newton), $f_{\text {class,branch }}(a 1$, newton $\left.)\right)$, so that the key constraint for class is violated.
We compensate this proliferation of atoms by introducing for $\mathcal{K}$ a new set inclusion relation $\subseteq \mathcal{K}$ (and based on that an equivalence relation $\equiv_{\mathcal{K}}$ ) that regards atoms as identical if the keys implies they are the same. With respect to this inclusion relation, the operator $C h_{\mathcal{K}}()$ is idempotent and monotonic. Moreover, for any constant tuple $\bar{c}$, query $Q$, and database instances $D, D^{\prime}$, possibly with Skolem terms, we have that $\bar{c} \in Q(D)$ implies $\bar{c} \in Q(D)$, whenever $D \subseteq \mathcal{K} D^{\prime}$. Then the proof of Theorem 2 can be generalized to the new setting, replacing set inclusion and equality with $\subseteq_{\mathcal{K}}$ and $\equiv_{\mathcal{K}}$.

2. Can be shown in the similar way as Claim 1 by generalizing the ideas in the proof of Theorem 2.

If the FKs are enforced, we also need to extend the available database obtained by applying $T_{\mathcal{C}}$ to the chase of $D_{Q}$ to satisfy $\mathcal{K}$. However, this cannot be done by chasing this result a second time. To see this, consider the query $Q_{\mathrm{sn}}$ from above and the TC statement $C_{p}=\operatorname{Compl}(\operatorname{pupil}(N, C, S)$; true $)$, which states that the table pupil is complete. Together with the enforced FK from pupil to class this implies completeness of $Q_{\mathrm{sn}}$, since then any available database must contain (i) all pupils and (ii) the class of each pupil. Let us try to reach this consequence by reasoning about the prototypical database for $Q_{\mathrm{sn}}$. Clearly, $D_{Q_{\mathrm{sn}}}=\{$ pupil(n,c,newton), class(c,newton, l, sci $)\}$ and $C h_{\mathcal{K}}\left(D_{Q_{\mathrm{sn}}}\right)=D_{Q_{\mathrm{sn}}}$, since there is already a class for the pupil in $D_{Q_{\mathrm{sn}}}$. Applying $T_{\mathcal{C}}$ yields $T_{\mathcal{C}}\left(C h_{\mathcal{K}}\left(D_{Q_{\text {sn }}}\right)\right)=\{$ pupil $(n, c$, newton $)\}$, since we are complete for pupil, but not for class. The chase would add a new class atom, say, class $\left(c\right.$, newton, $\left.l^{\prime}, b^{\prime}\right)$. However, $Q_{\text {sn }}$ will not retrieve the pupil $n$ over the database $\left\{\right.$ pupil( $(n, c$, newton $)$, class $\left(c\right.$, newton, $\left.\left.l^{\prime}, b^{\prime}\right)\right\}$, because it looks for pupils in a science class.

Clearly, instead of generating a new class atom, we should have copied the atom class $(c$, newton, $l, s c i)$ from the ideal $\mathrm{db}$ to satisfy the FK. To achieve this, we define the chase operator $C_{\mathcal{K}}^{a}(\cdot)$, which, given an incomplete database $\left(D^{i}, D^{a}\right)$ where $D^{i}$ satisfies $\mathcal{K}$, extends $D^{a}$ to satisfy $\mathcal{K}$ by copying atoms from $D^{i}$. Formally, if $R(\bar{s}) \in D^{a}$ and if $R[A] \subseteq S[B] \in \mathcal{K}$, we add all atoms $S(\bar{t}) \in D^{i}$ to $D^{a}$ where $S(\bar{t})[B]=R(\bar{s})[\bar{A}]$. The result is denoted as $C_{\mathcal{K}}^{a}\left(D^{i}, D^{a}\right)$.

To formalize the reasoning performed in the previous example, we combine the two chase operators and the $T_{\mathcal{C}}$ operator to a new operator $\mathcal{K}_{\mathcal{C}}$, defined as $\mathcal{K}_{\mathcal{C}}(D)=C_{\mathcal{K}}^{a}\left(C h(D), T_{\mathcal{C}}(C h(D))\right)$. This means that (i) we chase $D$ to obtain an ideal db that satisfies the foreign keys in $\mathcal{K}$, (ii) apply $T_{\mathcal{C}}$, and (iii) extend the result by copying atoms from $C h_{\mathcal{K}}(D)$.

THEOREM 7 (CHARACTERIZATION ENFORCED FKs). Let $\mathcal{C}$ be a set of TC statements, $\mathcal{K}$ be a weakly acyclic set of $F K s$, and $Q$ be a conjunctive query. Then

$$
\begin{aligned}
& \text { 1. } \mathcal{C} \models_{\mathcal{K}}^{e} \operatorname{Compl}^{s}(Q) \quad \Longleftrightarrow \quad \theta \bar{X} \in Q\left(\mathcal{K}_{\mathcal{C}}\left(D_{Q}\right)\right) \\
& \text { 2. } \mathcal{C} \models_{\mathcal{K}}^{e} \operatorname{Compl}^{b}(Q) \quad \Longleftrightarrow \quad D_{Q} \subseteq \mathcal{K}_{\mathcal{C}}\left(D_{Q}\right) .
\end{aligned}
$$

The proof generalizes the techniques for showing Theorem 6 . Now we extend our encoding so that it supports reasoning under FKs. Since a partial database satisfies an FK constraint if the ideal database satisfies it, we introduce for every FK constraint a rule like the one in (6), albeit for the ideal version of the relations involved. For instance, instead of (6), we introduce the rule $\operatorname{class}^{i}(C$, $\left.S, f_{\text {class, level }}(C, S), f_{\text {class, branch }}(C, S)\right) \leftarrow$ pupil $^{i}(N, C, S)$. We denote the set of all FK rules obtained from $\mathcal{K}$ as $P_{\mathcal{K}}^{i}$, where ${ }^{i}$ indicates that the rules create atoms for the ideal instance.

Finally, to model reasoning about enforced foreign keys, we introduce rules that copy foreign key atoms from the ideal to the available database. For example, the rule for the FK from pupil to class is

$$
\operatorname{class}^{a}(C, S, L, B) \leftarrow \operatorname{pupil}^{a}(N, C, S), \operatorname{class}^{i}(C, S, L, B) .
$$


We denote the set of all such rules obtained from $\mathcal{K}$ as $P_{\mathcal{K}}^{e}$, where $e^{e}$ indicates enforcement.

We are now ready to state the encoding theorems for reasoning about FKs, whose proofs are consequences of the Characterization Theorems 6 and 7.

TheOrem 8 (SET ENCOding FOR Non-ENFORCED FKS). Let $Q$ and $\mathcal{C}$ be as previously. Let $\mathcal{K}$ be a weakly acyclic set of $F K$ constraints. Then $\mathcal{C} \models_{\mathcal{K}} \operatorname{Compl}^{S}(Q)$ iff

1. the atom $Q^{s}$ is in the answer set of $P_{\mathcal{C}} \cup P_{\mathcal{K}}^{i} \cup P_{Q}^{s}$, or

2. $Q^{s}$ succeeds with $P_{\mathcal{C}} \cup P_{\mathcal{K}}^{i} \cup P_{Q}^{s}$ under SLD resolution.

Similar Encoding Theorems hold for any combination of set/bag semantics and non-enforced/enforced semantics. For bag semantics, we have to replace the rule set $P_{Q}^{S}$ by $P_{Q}^{b}$ and for enforced semantics we have to add the rule set $P_{\mathcal{K}}^{\mathcal{Q}}$.

\section{REASONING UNDER CONDITIONAL FINITE DOMAIN CONSTRAINTS}

Finite domain constraints make it necessary to reason by cases.

Example. We take up again the example from the introduction, with the query

$$
Q_{\mathrm{sn}}(N) \leftarrow \operatorname{pupil}(N, C, \text { newton }), \operatorname{class}(C, \text { newton }, L, s c i),
$$

asking for the pupils from the science branch at Newton school, the CFDC $F_{\mathrm{sn}}=$ class $\{$ school $=$ newton, branch $=s c i\}$ $[c$ code $]=\{a 1, b 2\}$, stating that the only science classes at Newton school are $a 1$ and $b 2$, and the TC statements $C_{\mathrm{a} 1 \mathrm{n}}=\operatorname{Compl}(\operatorname{pupil}(N$, al, newton $) ;$ true $)$ and $C_{\mathrm{b} 2 \mathrm{n}}=\operatorname{Compl}(\operatorname{pupil}(N, b 2$, newton $) ;$ true $)$, stating completeness for the pupils of classes $a 1$ and $b 2$. The TC statements alone do not entail completeness of $Q_{\mathrm{sn}}$, because in principle there could be other science classes. However, together with the CFDC, they do imply completeness.

The characterization in Theorem 2, though, does not apply here because the operators $T_{C_{\mathrm{a} 1 \mathrm{n}}}$ and $T_{\mathrm{C}_{\mathrm{b} 2 \mathrm{n}}}$ map the frozen body of $Q_{\mathrm{sn}}$ to the empty set. They transfer pupil atoms where the class code is $a l$ or $b 2$. Therefore, a reasoning procedure has to instantiate the codes in all ways permitted by the CFDC before applying the $T_{\mathcal{C}}$ operator. If the test of Theorem 2 succeeds for all such instantiations, then we can conclude completeness.

In the following, we develop concepts that allow us to characterize completeness wrt CFDCs and then provide an encoding of the characterizing condition. Since CFDCs are essentially disjunctive, we encode reasoning only into answer set programming, which supports disjunctive rules, while Prolog does not.

Let $\mathcal{F}$ be a fixed set of CFDCs. Suppose $B$ is a conjunction of atoms. Let $\gamma$ be a substitution that maps each variable $Y$ of $B$ either to a constant occurring in $\mathcal{F}$ (a proper constant) or to the frozen version of $Y$. We say that $\gamma$ satisfies $B$ and $\mathcal{F}$ if the instance $\gamma B$ satisfies $\mathcal{F}$. We say that a substitution $\gamma^{\prime}$ is at least as general as $\gamma$, written $\gamma^{\prime} \succ \gamma$, if for all variables $Y$ in $B$ and all proper constants $c$ we have that $\gamma^{\prime} Y=c$ implies $\gamma Y=c$. We say that $\gamma$ is an $\mathcal{F}$-case of $C$ if $\gamma$ is maximally general among all substitutions satisfying $B$ and $\mathcal{F}$. For instance, the body of the query $Q_{\text {sn }}$ has two $\left\{F_{\mathrm{sn}}\right\}-$ cases, $\gamma_{a l}$, which substitutes the variable $C$ with $a 1$ and freezes all other variables, and $\gamma_{b 2}$, which is analogously defined. By $\Gamma_{\mathcal{F}, B}$ we denote the set of all $\mathcal{F}$-cases of $B$.

We remark that by a reduction of the 3-colorability problem one can construct a set $\mathcal{F}_{3 \text { col }}$ of CFDCs such that it is NP-hard to decide whether $\Gamma_{\mathcal{F}_{3 \text { col }}, B} \neq \emptyset$ for a conjunction $B$. It is also not difficult to see that in polynomial time one can verify whether a substitution is an $\mathcal{F}$-case of $B$. Thus, checking whether $\Gamma_{\mathcal{F}, B} \neq \emptyset$ is NP-complete. We now generalize Theorem 2 to CFD constraints. We drop the analogous generalization of Theorem 4 due to the limited space.

THEOREM 9 (CHARACTERIZATION CFD CONSTRAINTS). Let $\mathcal{C}$ be a set of TC statements, $\mathcal{F}$ be a set of CFD constraints, and $Q(\bar{X}) \leftarrow B$ be a conjunctive query. Then

$$
\begin{aligned}
& \mathcal{C} \models_{\mathcal{F}} \operatorname{Compl}^{s}(Q) \Longleftrightarrow \\
& \gamma \bar{X} \in Q\left(T_{\mathcal{C}}(\gamma B)\right) \text { for every case } \gamma \text { in } \Gamma_{\mathcal{F}, B} .
\end{aligned}
$$

PROOF IDEA. For $\gamma \in \Gamma_{\mathcal{F}, B}$ we define the substitution $\tilde{\gamma}$ as $\tilde{\gamma} Y=$ $\gamma Y$ if $\gamma Y$ is a constant, and $\tilde{\gamma} Y=Y$ otherwise. That is, $\tilde{\gamma}$ substitutes variables that are subject to a CFD constraint by one of the possible values and leaves the other variables as they are. The proof then exploits the fact that over any database satisfying $\mathcal{F}$, a query $Q(\bar{X}) \leftarrow B$ is equivalent to the union of conjunctive queries $\bigcup_{\gamma \in \Gamma_{\mathcal{F}, B}} Q_{\gamma}$, where each $Q_{\gamma}$ is defined as $Q_{\gamma}(\tilde{\gamma} \bar{X}) \leftarrow \tilde{\gamma} B$.

Condition (7) stipulates a test comprising the following steps: (i) Instantiate the query body $B$ in all possible ways permitted by the CFD constraints. This amounts to considering all possible ways in which an answer to $Q$ can be retrieved. (ii) For each such instantiation $\gamma B$, compute $T_{\mathcal{C}}(\gamma B)$, the set of atoms that must be present in any available database if the ideal database contains $\gamma B$. (iii) Evaluate $Q$ over $T_{\mathcal{C}}(\gamma B)$ and check whether the result contains $\gamma \bar{X}$, the prototypical answer to $Q$ over $\gamma B$. We now show how to encode (i) $\mathcal{F}$-cases, (ii) the operator $T_{\mathcal{C}}$, and (iii) the final test into disjunctive rules.

The starting point is again the canonical database $D_{Q}$. Since $D_{Q}$ consists of ground atoms, which cannot be instantiated, we have to mimic the instantiation by cases. To this end, we introduce a new binary predicate $v a l$, where intuitively $\operatorname{val}(t, v)$ indicates that the term $t$ is instantiated by the value $v$. To achieve modularity, we keep the encodings of $Q, \mathcal{F}$, and $\mathcal{C}$ independent from each other. Therefore, no information as to which variable can be instantiated by which value should influence the encoding of $Q$. We achieve this by introducing $\operatorname{val}(t, t)$ for each term in $D_{Q}$ both for original constants and for frozen variables. The set of all such facts is denoted as $\mathrm{Val}_{Q}$. Then we require that every term can have at most one val-value other than itself, which can be seen as a requirement for $v a l$ to be functional. This can be expressed by the denial $r_{\text {fun }}$ :

$$
\leftarrow \operatorname{val}(X, Y), \operatorname{val}(X, Z), X \neq Y, X \neq Z, Y \neq Z .
$$

To mimic the instantiation of the query body by cases, we introduce for each CFD constraint $F=R\left\{a_{1}=v_{1}, \ldots, a_{n}=v_{n}\right\}[a]=\left\{w_{1}, \ldots\right.$, $\left.w_{m}\right\}$ the disjunctive rule $r_{F}$ as

$$
\begin{aligned}
& \operatorname{val}\left(X_{a}, w_{1}\right)|\cdots| \operatorname{val}\left(X_{a}, w_{m}\right) \leftarrow \\
& \quad R^{i}\left(X_{1}, \ldots, X_{a}, \ldots X_{n}\right), \operatorname{val}\left(X_{a_{1}}, v_{1}\right), \ldots, \operatorname{val}\left(X_{a_{n}}, v_{n}\right),
\end{aligned}
$$

which nondeterministically binds the term in attribute position $a$ to one of the values in $\left\{w_{1}, \ldots, w_{m}\right\}$, provided the attribute positions $a_{1}, \ldots, a_{n}$ are bound to $v_{1}, \ldots, v_{n}$, respectively. Thus, the CFD constraint $F_{\text {sn }}$ is translated into the rule $\operatorname{val}(C, a 1) \mid \operatorname{val}(C$, $b 2) \leftarrow \operatorname{class}(C, S, L, B), \operatorname{val}(S$, newton $), \operatorname{val}(B, \operatorname{sci})$. We collect the rules encoding $\mathcal{F}$ into the program $P_{\mathcal{F}}:=\left\{r_{F} \mid F \in \mathcal{F}\right\} \cup\left\{r_{\text {fun }}\right\}$.

We keep the rules $P_{\Sigma}$ that copy $D_{Q}$ into the ideal database $D_{Q}^{i}$. However, to make the TC rules applicable to facts with val-bindings, we need to unfold them. For an atom $A=R\left(t_{1}, \ldots, t_{n}\right)$, the unfolding consists of the atom $A^{u}=R\left(Y_{1}, \ldots, Y_{n}\right)$, where each argument is replaced by a fresh variable, and the set of val-bindings $U^{A}=\left\{\operatorname{val}\left(Y_{1}, t_{1}\right), \ldots, \operatorname{val}\left(Y_{n}, t_{n}\right)\right\}$. Earlier, we have shown how 
to translate a TC statement $C$ into the rule $r_{C}: R^{a}\left(t_{1}, \ldots, t_{n}\right) \leftarrow$ $R^{i}\left(t_{1}, \ldots, t_{n}\right), G^{i}$. The unfolded rule for $C$ is then

$$
\begin{aligned}
r_{C}^{u}: \quad R^{a}\left(Y_{1}, \ldots, Y_{n}\right) \leftarrow & R^{i}\left(Y_{1}, \ldots, Y_{n}\right), U^{R^{i}\left(t_{1}, \ldots, t_{n}\right)}, \\
& \left(G^{i}\right)^{u}, U^{G^{i}},
\end{aligned}
$$

where the unfolding $\left(G^{i}\right)^{u}$ of the condition $G^{i}$ and the set of value atoms $U^{G^{i}}$ are defined analogously to the case of single atoms. For example the rule $r_{C_{\mathrm{sci}}}^{u}$ for $C_{\mathrm{sci}}$ in (2) is

$$
\begin{aligned}
& \operatorname{pupil}^{a}\left(N_{1}, C_{1}, S_{1}\right) \leftarrow \\
& \operatorname{pupil}^{i}\left(N_{1}, C_{1}, S_{1}\right), \operatorname{val}\left(N_{1}, N\right), \operatorname{val}\left(C_{1}, C\right), \operatorname{val}\left(S_{1}, S\right), \\
& \operatorname{class}^{i}\left(C_{2}, S_{2}, L_{2}, B_{2}\right), \operatorname{val}\left(C_{2}, C\right), \operatorname{val}\left(S_{2}, S\right), \operatorname{val}\left(L_{2}, L\right), \operatorname{val}\left(B_{2}, \operatorname{sci}\right) .
\end{aligned}
$$

The program consisting of the unfolded rules is denoted as $P_{\mathcal{C}}^{u}=$ $\left\{r_{C}^{u} \mid C \in \mathcal{C}\right\}$.

Let $Q\left(X_{1}, \ldots, X_{n}\right) \leftarrow B$ be the query which we want to check for completeness. We have to modify the test query in two ways, first by unfolding, and second by adding $v a l$-atoms to encode the check whether $Q$ returns $\gamma \bar{X}$ over $T_{\mathcal{C}}(\gamma B)$. Thus, the rule $r_{Q_{s}^{u}}$ for the test query $Q_{s}^{u}$ is

$$
Q_{s}^{u} \leftarrow\left(B^{a}\right)^{u}, U^{B^{a}}, \operatorname{val}\left(x_{1}, X_{1}\right), \ldots, \operatorname{val}\left(x_{n}, X_{n}\right),
$$

where the $x_{j}$ are the frozen versions of the $X_{j}$. For example, the test query for $Q_{\mathrm{sn}}$ in (7) is

$$
\begin{aligned}
Q_{\mathrm{sn}}^{u} \leftarrow & \operatorname{pupil}^{a}\left(N_{1}, C_{1}, S_{1}\right), \operatorname{class}^{a}\left(C_{2}, S_{2}, L_{2}, B_{2}\right), \operatorname{val}\left(N_{1}, N\right), \\
& \operatorname{val}\left(n^{\prime}, N\right), \operatorname{val}\left(C_{1}, C\right), \operatorname{val}\left(S_{1}, \text { newton }\right), \operatorname{val}\left(C_{2}, C\right), \\
& \operatorname{val}\left(S_{2}, \text { newton }\right), \operatorname{val}\left(L_{2}, L\right), \operatorname{val}\left(B_{2}, \operatorname{sci}\right),
\end{aligned}
$$

where $n^{\prime}$ is the frozen version of $N$. Let $P_{Q}^{s, u}:=D_{Q}^{i} \cup \operatorname{Val}_{Q} \cup\left\{r_{Q_{s}^{u}}\right\}$ be the set of rules about $Q$.

THEOREM 10 (ASP ENCODING OF CFDCs). Let $Q$ be a conjunctive query, $\mathcal{C}$ a set of TC statements, and $\mathcal{F}$ a set of $C F D$ constraints. Then

$$
\begin{aligned}
& \mathcal{C} \models \mathcal{F} \operatorname{Compl}^{s}(Q) \Longleftrightarrow \\
& Q_{s}^{u} \text { is in every answer set of } P_{\mathcal{C}}^{u} \cup P_{\mathcal{F}} \cup P_{Q}^{s, u} .
\end{aligned}
$$

As in Theorem 5, we obtain an analogous encoding of bag completeness checks by modifying the rule for the test query. In order to ensure that no assignment is lost, we add to the body of the rule for $Q_{b}^{u}$ for every variable $Y$ in $Q$ an atom $\operatorname{val}(y, Y)$ with the frozen version $y$ of $Y$.

\section{FOREIGN KEYS AND CFDCS}

Foreign keys, in particular combined with conditional finite domains, allow for a larger set of inferences.

Example. Consider $Q_{\mathrm{n}}(N) \leftarrow \operatorname{pupil}(N, C$, newton $)$, which asks for the names of all pupils at Newton school, and assume the FK and CFD constraints from our running example hold. Consider as well the TC statement $C_{\text {sci }}$ in (2) and an analogous statement $C_{\text {hum }}$, which asserts completeness for pupils in humanities classes. Now, $Q_{\mathrm{n}}$ is complete because every pupil of Newton school belongs to a class of some branch (by the FKs), the only possible branches are $s c i$ and hum (by the CFDCs), and we are complete for each by $C_{\text {sci }}$ and $C_{\text {hum }}$. Note that we arrived at this conclusion without assuming that the FKs are enforced.

We now sketch how the constructions in Sections 5 and 6 can be combined to reasoning about CFDCs and FKs in conjunction. First we provide a characterization of completeness under set semantics.
The difference with respect to Theorem 9 is now that we have to consider all cases of the chase of the body $B$ of $Q$ instead of $B$ alone. One can show that it still does not matter which version of the chase is used.

THEOREM 11 (CHARACTERIZATION). Let $\mathcal{C}$ be a set of TC statements, $\mathcal{K}$ be a weakly acyclic set of $F K s, \mathcal{F}$ be a set of CFDCs, and $Q(\bar{X}) \leftarrow B$ be a conjunctive query. Then

$$
\begin{aligned}
\mathcal{C} & \models_{\mathcal{K}, \mathcal{F}} \operatorname{Compl}^{S}(Q) \Longleftrightarrow \\
\gamma \bar{X} & \Longleftrightarrow Q\left(T_{\mathcal{C}}\left(\gamma\left(\operatorname{Ch}_{\mathcal{K}}(B)\right)\right)\right) \text { for every case } \gamma \text { in } \Gamma_{\mathcal{F}, C h_{\mathcal{K}}(B)} .
\end{aligned}
$$

The characterization theorem for enforced FKs is a modification of Theorem 7 where $C h^{a}$ is to be applied to $\left(\gamma\left(C h_{\mathcal{K}}(B)\right)\right.$, $\left.T_{\mathcal{C}}\left(\gamma\left(C h_{\mathcal{K}}(B)\right)\right)\right)$ for every $\gamma$ in $\Gamma_{\mathcal{F}, C h_{\mathcal{K}}(B)}$.

For the encoding, there are now two modifications with respect to the case of plain FKs. The first is that every new term that is created by a chase rule must be a value of itself. The second is that the copy rules for enforced semantics need to be unfolded. For example, for the FK from pupil to class, we have to add the rule

$$
\operatorname{val}\left(f_{\text {class, level }}(C, S), f_{\text {class,level }}(C, S)\right) \leftarrow \operatorname{pupil}^{i}(N, C, S),
$$

which makes every new level term a value of itself. A similar rule is also needed for the branch of a class.

Let $\mathcal{K}$ be a set of FKs. We denote the extension of $P_{\mathcal{K}}$ by these rules as $P_{\mathcal{K}}^{u}$. Similarly, we denote the set of unfolded versions of the copy rules as $P_{\mathcal{K}}^{e, u}$. With these rules we can now encode completeness reasoning that considers both FKs and CFDCs into answer set programming.

Theorem 12 (ASP Encoding). Let $Q, \mathcal{C}$, and $\mathcal{F}$ as before and let $\mathcal{K}$ be a weakly acyclic set $F K$ constraints. Then

$$
\begin{aligned}
& \text { 1. } \mathcal{C} \models_{\mathcal{F}, \mathcal{K}} \operatorname{Compl}^{s}(Q) \Longleftrightarrow \\
& Q_{s}^{u} \text { is in every answer set of } P_{\mathcal{C}}^{u} \cup P_{\mathcal{F}} \cup P_{\mathcal{K}}^{i, u} \cup P_{Q}^{s, u} ; \\
& \text { 2. } \mathcal{C} \models_{\mathcal{F}, \mathcal{K}}^{e} \operatorname{Compl}^{s}(Q) \Longleftrightarrow \\
& Q_{s}^{u} \text { is in every answer set of } P_{\mathcal{C}}^{u} \cup P_{\mathcal{F}} \cup P_{\mathcal{K}}^{i, u} \cup P_{\mathcal{K}}^{e, u} \cup P_{Q}^{s, u} .
\end{aligned}
$$

An analogous result holds for bag semantics.

\section{EXPERIMENTS}

We performed experiments to find out what is the maximal size of problems that can be solved with our encodings on standard Prolog and Answer Set Programming (ASP) platforms and how the running time depends on the input. Since we expect that in applications there are many constraints that are not relevant to a reasoning problem, we also explored how the performance is influenced by irrelevant information.

We performed four tests and ran them on two state-of-the-art ASP reasoners, dlv and clingo. We also ran the first test on the swi Prolog implementation. The experiments were executed on a desktop machine with an Intel i5 3.40GHz CPU and 8GB memory. Each experiment was repeated ten times and since the time measurements were close, we show here average running times.

For the tests, we extended our schema with two relations: school (sname, scluster,type) and schoolcluster(scluster, district, status), which model that every school belongs to some school cluster and is of a certain type, e.g., school(newton, cluster5, middle), and that each school cluster is in some district and has the status public or private, e.g., schoolcluster(cluster5, north,pub). 
Test 1. We studied the cost of reasoning with FKs with the query

$$
\begin{aligned}
Q_{1 \mathrm{pub}}(N) \leftarrow & \operatorname{pupil}(N, C, S), \operatorname{class}(C, S, 1, B), \\
& \operatorname{school}(S, S C, T), \text { schoolcluster }(S C, D, p u b),
\end{aligned}
$$

which asks for "all $1^{\text {st }}$ level pupils from public clusters." We asserted that we are complete for pupils, expressed by pupil ${ }^{a}(N, C$, $S) \leftarrow \operatorname{pupil}^{i}(N, C, S)$, and that we enforced the FKs pupil[ccode, sname $] \subseteq$ class [ccode, sname], class $[$ sname $] \subseteq \operatorname{school}[$ sname $]$, and school[scluster $] \subseteq$ schoolcluster $[$ scluster $]$. So, $Q_{1 \text { pub }}$ is complete under these assertions.

To simulate reasoning in the presence of irrelevant information, we added TC statements of the form

$$
\operatorname{pupil}^{a}(N, \$ c, \$ s) \leftarrow \operatorname{pupil}^{i}(N, \$ c, \$ s),
$$

where $\$ c$ ranged over $\left\{1,10,10^{2}\right\}$ and $\$ s$ ranged over $\{1,10, \ldots$, $\left.10^{5}\right\}$, which gave rise to 8 test cases with $10^{0}$ to $10^{7}$ irrelevant TC statements.

The results in Figure 1 show that all systems could deal with sets of up to $10^{6} \mathrm{TC}$ statements. While swi Prolog could deal also with the largest input, the ASP reasoners timed out after 30 minutes. Moreover, for small and large sets of TC statements, swi was one order of magnitude faster than the ASP reasoners.

Test 2. We wanted to assess the cost of reasoning with CFDCs. We ran this test only on ASP reasoners, since implementing CFD reasoning in Prolog requires the usage of meta-programming predicates to cope with disjunctive rules. We reasoned about the query

$$
\begin{aligned}
Q_{\text {high }}(N) \leftarrow & \operatorname{pupil}(N, C, S), \operatorname{class}(C, S, L, B), \\
& \operatorname{school}(S, S C, \text { high }),
\end{aligned}
$$

which asks for "all pupils from a class at a high school."

We enumerated all possible schools, class levels, and class branches with FDCS of the form class \{\}$[$ sname $]=\left\{s_{1}, \ldots, s_{k}\right\}$, class \{\}$[$ level $]=\left\{l_{1}, \ldots, l_{m}\right\}$, and class \{\}$[$ branch $]=\left\{b_{1}, \ldots, b_{n}\right\}$. In addition, we asserted that we have complete information about all schools, Compl(school $(S, S C, T)$; true), all school classes, Compl(class $(C, \$ S, L, B)$; true), and all pupils in all classes, Compl $\operatorname{pupil}(N, C, \$ S) ; \operatorname{class}(C, \$ S, \$ L, \$ B))$, where $\$ S, \$ L$, and $\$ B$ range over all school names, class levels, and branches, resp. The combination of the CFD constraints and the TC statements entail that $Q_{\text {high }}$ is complete. To conclude completeness, all possible cases combining school name, class level, and branch must be explored. We varied the sizes of these three domains in such a way that the number of cases (and the number of TC statements for class) ranged from 1 to $10^{6}$.

From the results in Figure 2 we observe that for both $\mathrm{Clingo}$ and $\mathrm{dlv}$ the running times were linear in the size of the input. Both reasoners could cope with up to 1 mio cases. The fastest reasoner, $\mathrm{dl} v$, could process $10^{4}$ cases within less than 1 second, which we deem acceptable.

Test 3. We wanted to check whether the running time observed in Test 2 depended on the size of the input or on the number of cases. We constructed an extreme setup where we had a small input, but a large number of cases, and where completeness of the query followed already from few TC statements, while the case analysis was not needed to conclude completeness. We reasoned about the query

$$
\begin{aligned}
Q_{\text {pub }}(N) \leftarrow & \operatorname{pupil}(N, C, S), \operatorname{class}(C, S, L, B), \\
& \operatorname{school}(S, S C, T), \operatorname{schoolcluster}(S C, D, p u b),
\end{aligned}
$$

which asks for "all pupils from public clusters," in the presence of TC statements that assert the completeness of each of the relations

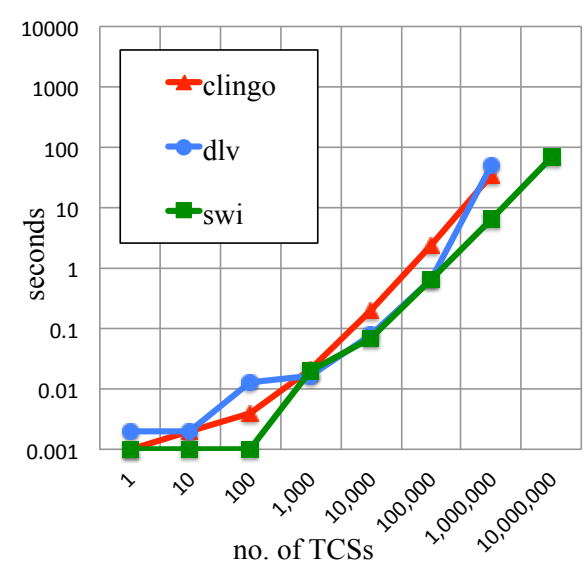

Figure 1: Test 1

in the query, such as $\operatorname{Compl}(\operatorname{pupil}(N, C, S)$; true $)$, etc. Together, these TCSs guaranteed the completeness of $Q_{\text {pub }}$.

Additionally, we asserted a chain of CFDCs that did not contribute to the completeness of $Q_{\text {pub }}$, but made the reasoning more complicated. Specifically, we stated that there are $i$ different districts, schoolcluster $[$ district $]=\left\{d_{1}, \ldots, d_{i}\right\}$, that for every such district $d$ there are $j$ different public school clusters, schoolcluster $\{$ district $=d$, status $=p u b\}[$ scluster $]=\left\{s c_{1}, \ldots, s c_{j}\right\}$, and that for each such cluster there are $m$ different schools, while for each school, there are $n$ different levels.

We varied the parameters $i, j, m, n$ and $k$ to be 1 or 10 ( $i$ could be also 100) to obtain different sizes of CFDCs, which gave rise to problems with $10^{p}$ answer sets, where $p=0, \ldots, 6$.

Figure 3 displays the running times as depending on the number of answer sets, instead on the input size, which is logarithmically smaller. We observed running times linear in the number of answer sets, which correspond to the number of possible cases, but exponential in the size of the input. The running time, measured in this way, is approximately 10 times faster, than in Test 2, where the case analysis was actually needed. We conclude that reasoning about irrelevant cases takes time, but less than about relevant cases.

Test 4. We wanted to assess the cost of reasoning jointly about FKs and CFDCs. To this end, we slightly modified the setup of Test 3: (i) we dropped the TC statement asserting the completeness of class, and (ii) added the enforced FK constraint pupil [ccode, sname $] \subseteq$ class $[$ ccode, sname $]$, which ensures the presence of all class information needed for the query.

The results in Figure 4 show that the number of answer sets that reasoners could cope with before the 30 minutes timeout dropped from $10^{6}$ to $10^{4}$ and that the running time was no more linear in the number of cases.

In summary, our encoding of completeness reasoning allows for efficient reasoning with foreign keys in the presence of large numbers of TC statements, while reasoning about CFDCs depends in the first place on the number of cases covered by a set of CFDCs, rather than the input size. For combinations of FKs and CFDCs, the performance is not yet fully satisfactory.

\section{CONCLUSION}

We presented techniques to reason about query completeness over partially complete databases satisfying primary and foreign key constraints as well as conditional finite domain constraints. This generalizes previous work on query completeness and allows 


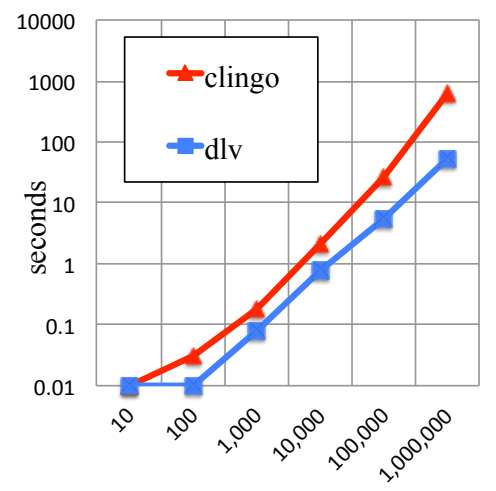

no. of answer sets (no. of TCSs)

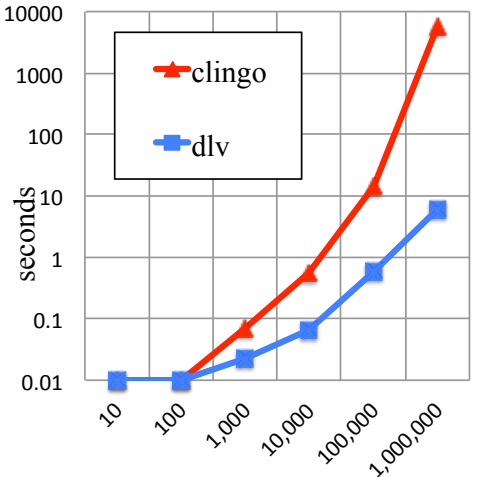

no. of answer sets

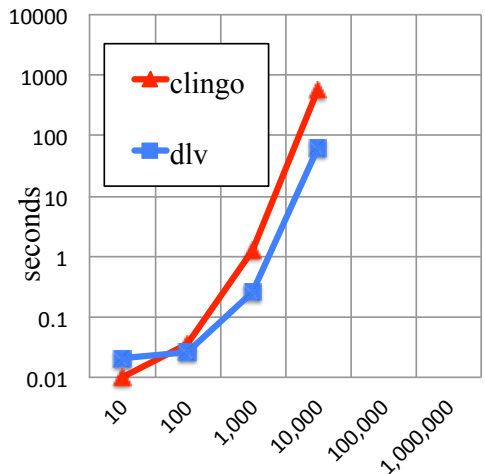

no. of answer sets

Figure 2: Test 2

Figure 3: Test 3

one to identify more situations in which queries are complete than was possible before. Our approach also provides a basis for the implementation of these reasoning techniques. We provided syntactic characterizations of completeness reasoning tasks which are independent of any implementation. In a second step, we used them to develop provably correct encodings into logic programming, with and without disjunctive rules, depending on the constraints involed. The encodings can be executed immediately by Prolog engines or ASP solvers.

As a proof of concept, we tested these encodings in a scenario that reflects the requirements of an administrative information system. The results suggest that the encodings are adequate for medium-sized data sets, as they may occur in the administration of a cluster of schools or a university. Currently, we are working on the integration of completeness management into the research information system of a university.

Acknowledgments. This work was partially supported by the project "Managing Completeness of Data (MAGIC)", funded by the province of Bolzano, and the project "Completeness-Aware Querying and Navigation on the Web of Data (CANDy), funded by the Free University of Bozen-Bolzano." We thank the anonymous reviewers for their helpful comments.

\section{REFERENCES}

[1] S. Abiteboul, R. Hull, and V. Vianu. Foundations of Databases. Addison-Wesley, 1995.

[2] K. Apt and M. van Emden. Contributions to the theory of logic programming. J. ACM, 29(3):841-862, 1982.

[3] C. Baral. Knowledge Representation, Reasoning, and Declarative Problem Solving. Cambridge University Press, New York, NY, USA, 2003.

[4] A. Chandra and P. Merlin. Optimal implementation of conjunctive queries in relational databases. In Proc. 9th STOC, 1977.

[5] A. Cortés-Calabuig, M. Denecker, O. Arieli, and M. Bruynooghe. Approximate query answering in locally closed databases. In AAAI, pages 397-402, 2007.

[6] A. Cortés-Calabuig, M. Denecker, O. Arieli, and M. Bruynooghe. Accuracy and efficiency of fixpoint methods for approximate query answering in locally complete databases. In $K R$, pages 81-91, 2008.

[7] R. Fagin, P. G. Kolaitis, R. J. Miller, and L. Popa. Data exchange: Semantics and query answering. In ICDT 2003, pages 207-224, 2003.
[8] W. Fan and F. Geerts. Relative information completeness. In PODS, pages 97-106, 2009.

[9] W. Fan and F. Geerts. Capturing missing tuples and missing values. In PODS, pages 169-178, 2010.

[10] M. Gebser, B. Kaufmann, A. Neumann, and T. Schaub. clasp: A conflict-driven answer set solver. In In LPNMR '07, pages 260-265. Springer, 2007.

[11] M. Gelfond and V. Lifschitz. Classical negation in logic programs and disjunctive databases. New Generation Computing, 9:365-385, 1991.

[12] D. S. Johnson and A. C. Klug. Testing containment of conjunctive queries under functional and inclusion dependencies. In PODS, pages 164-169, 1982.

[13] A. Klug. On conjunctive queries containing inequalities. $J$. ACM, 35(1):146-160, 1988.

[14] N. Leone, G. Pfeifer, W. Faber, T. Eiter, G. Gottlob, S. Perri, and F. Scarcello. The DLV system for knowledge representation and reasoning. ACM Trans. Comput. Log., 7(3):499-562, 2006.

[15] A. Levy. Obtaining complete answers from incomplete databases. In Proc. VLDB, pages 402-412, 1996.

[16] B. Marnette. Generalized schema-mappings: from termination to tractability. In PODS, pages 13-22, 2009.

[17] A. Motro. Integrity = Validity + Completeness. ACM TODS, 14(4):480-502, 1989.

[18] S. Paramonov. Query completeness-A logic programming approach. Technical Report KRDB13-2, KRDB Research Center, Free Univ. Bozen-Bolzano, 2013. http://www.inf.unibz.it/krdb/pub/tech-rep.php.

[19] S. Razniewski and W. Nutt. Checking query completeness over incomplete data. Technical Report KRDB11-2, KRDB Research Center, Free University of Bozen-Bolzano, 2011.

[20] S. Razniewski and W. Nutt. Completeness of queries over incomplete databases. In $V L D B, 2011$.

[21] O. Savković, M. Paramita, S. Paramonov, and W. Nutt. MAGIK: Managing completeness of data. In CIKM, pages 2725-2727, 2012.

[22] O. Savković, M. Paramita, A. Tomasi, and W. Nutt. Complete approximations of incomplete queries. $P V L D B$, 6(12):1378-1381, 2013.

[23] P. Simons, I. Niemelá, and T. Soininen. Extending and implementing the stable model semantics. Artif. Intell., 138(1-2):181-234, June 2002. 